\title{
Prevalence of central line associated blood stream infection (CRBSI) and catheter colonization in ICU settings of a tertiary care hospital in sub Himalayan region
}

\author{
Negi N. ${ }^{1}$, Gupta S. ${ }^{2}$, Chandola I. ${ }^{3}$, Kataria V. ${ }^{4}$ \\ ${ }^{1}$ Dr. Nidhi Negi, ${ }^{2}$ Ms. Suchandra Gupta, ${ }^{3}$ Dr. Iva Chandola, ${ }^{4}$ Dr. Vijay Kataria, all authors are affiliated with Department \\ of Microbiology, Sri Guru Ram Rai Institute of Medical and Health Sciences, Patel Nagar, Dehradun, Uttarakhand, India.
}

Corresponding Author: Dr. Iva Chandola, Department of Microbiology, Sri Guru Ram Rai Institute of Medical and Health Sciences, Patel Nagar, Dehradun, Uttarakhand, India, Email: chandolaiva@gmail.com

\begin{abstract}
Background: Catheter-related bloodstream infections (CRBSIs) are one of the major causes of morbidity and mortality in critically ill hospitalized patients mainly in ICU settings. Emergence of multidrug resistant bacteria makes it compulsory to know the current sensitivity profile of the organism against antimicrobial agents. Objective: To isolate the etiological agents responsible for CRBSI and to find out the spectrum of antimicrobial sensitivity of isolated organisms. Material and Methods: A total of 180 suspected cases of CRBSI were included in the study in which 44 (24.44\%) cases were positive for catheter tip colonization. Further isolation was done using standard laboratory procedures. Result: Out of a total of 180 suspected cases of CRBSI 44 cases were found to be positive for catheter tip colonization after which they were divided into two groups 4 (2.2\%) had CRBSI while $40(22.22 \%)$ had local catheter infections. Conclusion: Catheter colonization and duration of catheterization has an important role in development of CRBSI which may lead to septicaemia and multi-organ failure. CRBSI must be suspected in the catheterized patient having sign and symptoms of septicaemia.
\end{abstract}

Keywords: Central Venous Catheter (CVC), Septicaemia, CVC colonization.

\section{Introduction}

Catheter-related bloodstream infection (CRBSI) is defined as the presence of bacteremia which originates from an indwelling intravenous catheter. It is one of the most lethal, and costly complications of central venous catheterization [1]. It is also the most common cause of healthcare associated infections like bacteremia and septicemia. These result after diffusion of bacteria in blood from a localized source (secondary BSI) or may be the only identifiable infectious process (primary BSI). ICUs remains foci of these infections mainly because they cater group of patients who are mostly immuno-compromised and are at increased risk of becoming infected after undergoing various invasive therapeutic and diagnostic procedures $[2,3,4]$.

Central venous catheters (CVC) are mainly used in critically ill patients for the administration of medications, fluids, nutritional solutions and blood products [5]. It has been observed that use of CVCs

Manuscript received: $6^{\text {th }}$ October 2019

Reviewed: $16^{\text {th }}$ October 2019

Author Corrected: $20^{\text {th }}$ October 2019

Accepted for Publication: $23^{\text {rd }}$ October 2019 plays a major role in developing CRBSI and catheter related local infections (CRLI) as well, which has become a greater cause of morbidity and mortality. Catheter related infections are of two types: Catheter related local infections (CRLI) which can be defined as the presence of catheter tip colonization and any sign of local infection which includes in duration, erythema, heat, pain or purulent drainage. Catheter related blood stream infection (CRBSI) can be defined as positive blood culture from a peripheral vein with signs of systemic infection (fever, chills) with no other source of bacteremia but with the same organism isolated from catheter and catheter tip colonization [6].

Both Gram positive and Gram negative organisms are associated with blood stream infections. When bacteria multiply and release their toxins in blood stream, it activates the production of cytokines, leading to fever, chills, toxicity and shock [4]. Numerous risk factors are associated with CRBSI like duration of catheterization, type of catheter, site of catheter insertion, number of lumens, location of patient, pressure of needle less

Pathology Update: Tropical Journal of Pathology \& Microbiology Available online at: www.medresearch.in 815 | P a g e 


\section{Original Research Article}

connectors and whether proper care practices are being followed or not. laboratory confirmation of central venous catheter infection requires recovery of the same organism from the patient's blood as well as from cultures of the catheter tip or intracuatneous catheter segment. Hence it becomes necessary to submit both catheter segments and blood culture to the laboratory for the diagnosis of catheter related blood stream infections. Several methods have been used to diagnose catheter related blood stream infections. Semiquantitative (roll plate) cultures, catheter flush cultures and quantitative (sonication) cultures are more reliable diagnostic methods than qualitative cultures where the tip is culture in broth and single contaminating microbe can give a positive result $[7,8]$.

Semiquantitative technique is reported to distinguish infection from contamination, with counts of $>15 \mathrm{CFU}$ considered significant [9]. The quantitative method and flush culture method use $>10^{2} \mathrm{CFU}$ as the significant count for most organisms. However lower counts of particular organisms, including $S$. aureus and Candida albicans may be clinically significant if there is associated bacteremia [10].

In this study, incidence of CVC colonization, incidence of CRBSIs, various organisms related to CRBSI and CRLIs were focused into along with their antimicrobial susceptibility pattern and association between colonization of catheter and CRBSI in our ICU setup.

\section{Materials and methods:}

The study was conducted in Shri Guru Ram Rai Institute of Medical and Health Sciences Patel Nagar, Dehradun, from July 2017 to June 2018. A total of 180 patients admitted to the various wards and ICUs of hospital requiring catheterization and having sign and symptoms of septicaemia after 48 hours of central venous catheter insertion were included in this study.

Catheter tip for culture was accepted only if there were any of the signs of infection like Inflammation at the insertion site, fever, sign of sepsis, documented bacteremia in which the source is not apparent. Patients with any obvious source of infections and having infective endocarditis, retroviral disease and immunosuppressive drugs, catheter put outside our hospital, urinary foley catheter tips, chest tube tips, abdominal drainage tips, tips placed in saline or transport medium were not accepted. Semiquantitative (roll-plate) method was used in which distal $5 \mathrm{~cm}$ catheter was cut with sterile blade and was kept in a sterile container. Using sterile forceps, catheter tip was removed from transport tube and was laid on Blood agar plate and MacConkey agar plate. The tip was rolled back and forth across the entire surface of a Blood agar plate using sterile forceps and exerting slight downward pressure. Plates were incubated at $37^{\circ} \mathrm{C}$ under aerobic conditions and read at 24 and 48hours. Count was done of each type of colony isolated, comparing growth on each medium. Each organism which was present from vascular catheter tips with colony counts of $\geq 15 \mathrm{CFU}$, including Grampositive rods were identified.

For counts of $\leq 15 \mathrm{CFU}$, only significant pathogens (e.g Candida albicans, group-A Streptococci and Gramnegative rods) were identified ${ }^{3}$.Plates were saved for 1 week to compare with growth in Blood culture. A set of paired blood culture were collected from peripheral sites. Blood culture bottles was placed in Bact/Alert 3D Automated Blood Culture system (BioMérieux).

From positive blood culture bottles, few drops of media was taken after gentle agitation and was subcultured on blood agar and Mac Conkey agar plates and were incubated overnight at $37^{\circ} \mathrm{C}$. Antimicrobial susceptibility was done by automated method VITEK 2. For reporting of the laboratory results following definitions were followed:

Catheter related blood stream infection: A positive blood culture with same organism isolated from catheter tip culture was considered to indicate CRBSI.

Catheter Colonization: A positive quantitative catheter culture with $>1000 \mathrm{cfu} / \mathrm{ml}$ was considered as catheter colonization.

Blood stream infection: Positive blood culture with negative Catheter tip culture or different organism isolated from Catheter tip was presumed to be blood stream infection (cause may be other than catheter) [11].

\section{Results}

In the present study, catheters were inserted for duration that ranged from 7 to 45 days with a mean of 21 days and a total of 1396 days. A total of 180 cases were included in the study in which 44 cases were positive for catheter tip colonization.

Out of 44 cases they were divided into two groups 4 had CRBSI while 40 had local catheter infections.

Pathology Update: Tropical Journal of Pathology \& Microbiology Available online at: www.medresearch.in 816 | P a g e 
Table 1 shows Demographic profile of the subjects in which number of cases are maximum (38\%) in 40-60 years of age group followed by 21-40 years of age group (27.7\%).

Table No.-1 (a): demographic profile of subjects

\begin{tabular}{|c|c|}
\hline Range in Years & Number of Cases (n=180) (\%) \\
\hline $0-20$ & $20(11.1 \%)$ \\
\hline $21-40$ & $50(27.7 \%)$ \\
\hline $41-60$ & $70(38.8 \%)$ \\
\hline Above 60 & $40(22.2 \%)$ \\
\hline
\end{tabular}

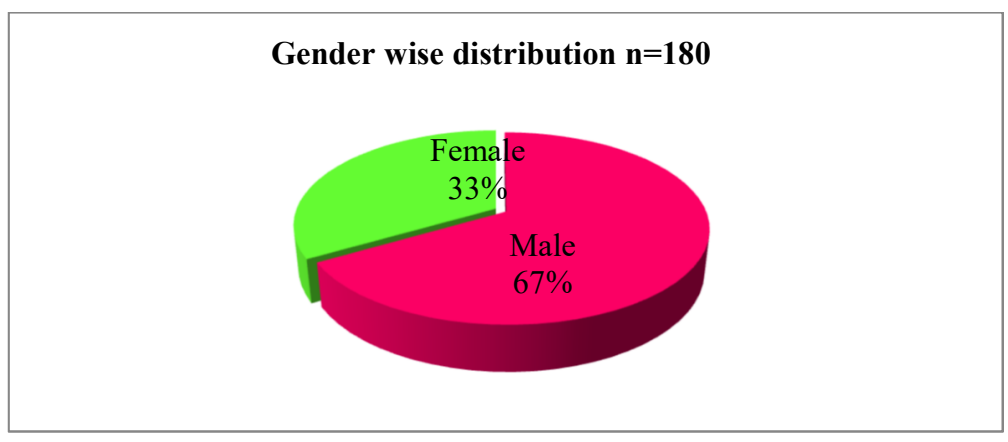

Fig.-1: Gender wise distribution.

Table-2: Profile of organisms isolated from catheter tip colonization

\begin{tabular}{|l|c|}
\hline Organisms & Total number of isolates (n=44) \\
\hline Serratia marcescens & $7(17.5 \%)$ \\
\hline Klebsiella pneumoniae & $6(15.0 \%)$ \\
\hline Candida albicans & $6(15.0 \%)$ \\
\hline Enterobacter cloacae & $5(12.5 \%)$ \\
\hline Proteus mirabilis & $3(7.5 \%)$ \\
\hline Acinetobacter baumanii & $3(7.5 \%)$ \\
\hline Pseudomonas aeuroginosa & $3(7.5 \%)$ \\
\hline Non albicans candida & $2(5.0 \%)$ \\
\hline Escherichia coli & $2(5.0 \%)$ \\
\hline Citrobacter species & $2(5.0 \%)$ \\
\hline Streptococcus agalactiae & $1(2.5 \%)$ \\
\hline
\end{tabular}

According to Table 2, rate of CVC colonization was $22.22 \%(40 / 180)$ which includes maximum number from family Enterobacteriaceae i.e. $62.5 \%(25 / 40)$ followed by yeast $20 \%(8 / 40)$ and non-fermenter group $15 \%(6 / 40)$.

Table-3 (a): Catheter profile of the study population

\begin{tabular}{|l|c|}
\hline Catheter profile & Number of cases \\
\hline a) Type of catheter & 44 \\
\hline Central venous catheter & 0 \\
\hline b) Number of lumen & 44 \\
\hline Single & 0 \\
\hline Double & \\
\hline Triple & 30 \\
\hline c) Site of venous cannulation & 14 \\
\hline Right Subclavian vein & 0 \\
\hline Left subclavian vein & 0 \\
\hline Juglar vein & \multicolumn{2}{|c|}{} \\
\hline Femoral vein & 0 \\
\hline
\end{tabular}




\section{Original Research Article}

Table-3(b): Clinical Profile of Subjects

\begin{tabular}{|l|c|c|}
\hline Clinical profile & $\begin{array}{c}\text { Catheter related blood stream infections } \\
\text { (CRBSI) n=4 }\end{array}$ & $\begin{array}{c}\text { Catheter tip } \\
\text { colonization n }=44\end{array}$ \\
\hline Gender & $3(75 \%)$ & $28(70 \%)$ \\
\hline Males & $1(25 \%)$ & $16(30 \%)$ \\
\hline Females & $1(25 \%)$ & $15(34 \%)$ \\
\hline Morbidity associated & $4(100 \%)$ & $34(77.2 \%)$ \\
\hline Altered liver function test & $0(0 \%)$ & $04(9.09 \%)$ \\
\hline Altered renal function test & & $36(81.8 \%)$ \\
\hline Altered coagulation profile & $4(100 \%)$ & $27(61.3 \%)$ \\
\hline Hematologic dysfunction: & $2(50 \%)$ & $26(59.09 \%)$ \\
\hline Low Hb & $3(75 \%)$ & \\
\hline High ESR & & \\
\hline High TLC & & \\
\hline
\end{tabular}

As per Table 3a, clinical profile and catheter characteristics of the study population have been shown from where it is interpreted that central venous catheters were used with double lumen. Most common site of insertion was right subclavian vein 30 (68\%) followed by left subclavian vein 14 (32\%). Table $3 \mathrm{~b}$ describes the clinical profile of both types of patients with CRBSI and CRLIs.

All patients with CRBSI were having altered kidney function (100\%) and low Hb count (100\%) while none of them were having altered coagulation profile. Whereas in case of patients associated with catheter tip colonization altered kidney function was associated in $77.2 \%$ cases and low $\mathrm{Hb}$ count was seen in $81.8 \%$ cases. $34 \%$ cases were associated with altered liver function and $9 \%$ cases were associated with altered coagulation profile.

Table-4: Organisms isolated from catheter related blood stream infection (CRBSI)

\begin{tabular}{|l|c|}
\hline Name of isolates & Number of isolates (n=4) \\
\hline Klebsiella pneumonia & $1(25 \%)$ \\
\hline Enterobacter cloacae & $1(25 \%)$ \\
\hline Candida albicans & $1(25 \%)$ \\
\hline Non albicans candida & $1(25 \%)$ \\
\hline
\end{tabular}

Isolates obtained in CRBSI i.e. same organism isolated from catheter tip as well as blood culture are mentioned in Table 4.

Table-5: Resistance pattern of Enterobacteriaceae members isolated from catheter tip colonization:

\begin{tabular}{|l|c|c|c|c|c|}
\hline Antibiotic & $\begin{array}{c}\text { S. marcescens } \\
(\mathbf{n = 7})\end{array}$ & $\begin{array}{c}\text { K.pneumoniae } \\
(\mathbf{n = 6 )}\end{array}$ & $\begin{array}{c}\text { E. cloacae } \\
(\mathbf{n}=\mathbf{5})\end{array}$ & $\begin{array}{c}\text { P. mirabilis } \\
(\mathbf{n = 3})\end{array}$ & $\begin{array}{c}\text { E.coli } \\
(\mathbf{n}=\mathbf{2})\end{array}$ \\
\hline Amikacin & $7 / 7(100 \%)$ & $6 / 6(100 \%)$ & $0 / 5(0 \%)$ & $0 / 3(0 \%)$ & $1 / 2(50 \%)$ \\
\hline $\begin{array}{l}\text { Amoxicillin } \\
\text { clavulenic acid }\end{array}$ & $0 / 7(0 \%)$ & $4 / 6(67 \%)$ & $2 / 5(40 \%)$ & $0 / 3(0 \%)$ & $2 / 2(100 \%)$ \\
\hline Ceftriaxone & $0 / 7(0 \%)$ & $5 / 6(83 \%)$ & $0 / 5(0 \%)$ & $0 / 3(0 \%)$ & $2 / 2(100 \%)$ \\
\hline Ciprofloxacin & $7 / 7(100 \%)$ & $5 / 6(83 \%)$ & $0 / 5(0 \%)$ & $0 / 3(0 \%)$ & $1 / 2(50 \%)$ \\
\hline Colistin & $7 / 7(100 \%)$ & $2 / 6(33 \%)$ & $0 / 5(0 \%)$ & $3 / 3(100 \%)$ & $0 / 2(0 \%)$ \\
\hline Imipenem & $0 / 7(0 \%)$ & $6 / 6(100 \%)$ & $0 / 5(0 \%)$ & $2 / 3(67 \%)$ & $0 / 2(0 \%)$ \\
\hline $\begin{array}{l}\text { Piperacillin-s } \\
\text { Tazobactam }\end{array}$ & $0 / 7(0 \%)$ & $6 / 6(100 \%)$ & $0 / 5(0 \%)$ & $0 / 3(0 \%)$ & $1 / 2(50 \%)$ \\
\hline Tigecyclin & $0 / 7(0 \%)$ & $0 / 6(0 \%)$ & $0 / 5(0 \%)$ & $2 / 3(67 \%)$ & $0 / 2(0 \%)$ \\
\hline
\end{tabular}


Original Research Article

Table-6: Resistance pattern of non-fermenters isolated from CRLI

\begin{tabular}{|c|c|c|}
\hline Antibiotics & $\begin{array}{c}\text { P.aeuroginosa } \\
(\mathbf{n = 3})\end{array}$ & $\begin{array}{c}\text { A.baumanii } \\
(\mathbf{n}=3)\end{array}$ \\
\hline Amikacin & $3 / 3(100 \%)$ & $3 / 3(100 \%)$ \\
\hline Ciprofloxacin & $3 / 3(100 \%)$ & $0 / 3(0 \%)$ \\
\hline Colistin & $1 / 3(33 \%)$ & $1 / 3(33 \%)$ \\
\hline Imipenem & $3 / 3(100 \%)$ & $3 / 3(100 \%)$ \\
\hline Tigecyclin & $2 / 3(67 \%)$ & $0 / 3(0 \%)$ \\
\hline PipTaz & $1 / 3(33 \%)$ & $3 / 3(100 \%)$ \\
\hline
\end{tabular}

Anitibiotic sensitivity profile was done in all patients with Catheter related blood stream infections. Enterobacter cloacae and Serratia merscens were $100 \%$ sensitive for Amoxycillin clavulanic acid, Ceftriaxone and Imipenem while Acinetobacter baumanii were 100\% sensitive for Ciprofloxacin and Tigecyclin. Drug resistance were observed in the patients with catheter related local infections especially Klebsiella pneumoniae, Pseudomonas aeuroginosa and Acinetobacter baumanii were $100 \%$ resistant for Amikacin, and Imipenem.

\section{Discussion}

Intravascular catheters mainly those which have access to great vessels of our body i.e. central line catheters, are of essential use in health care settings especially in intensive care units. Such catheters provide necessary vascular access but their use puts patients at risk for local and systemic infectious complications, including local site infection and catheter-related bloodstream infections (CRBSI).

In the present study only CVC colonization rate was $24.4 \%$, Gahlot et al [5] found in their study that CVC colonization rate was $62.5 \%$. In present study it was observed that the commonest isolates responsible for causing Catheter tip colonisation belongs to Enterobacteriaceae (62.5\%) among which most predominant organism was S.mersescens, $K$. pneomoniae followed by yeast $(20 \%)$ and nonfermenters (15\%). Parmeswaran et al. [12] from Karnataka found commonest isolates associated with Catheter tip colonization were Gram negative (61.3\%), Gahlot et al. from Varanasi observed that Gram positive cocci most common (40\%) followed by Gram negative non-fermenters (26.8\%). Subba Rao et al. from Karnataka [2] showed that commonest isolates in ICU patients were CONS (32.4\%).

In the present study rate of CRBSI was $2.2 \%$ (4/180) per 1000 catheter days. Pawar et al [13] showed rate of CRBSI in their study was $4.01 \%$. Parmeshwaram et al [12] found CRBSI rate in their study was $8.75 \%$ per catheter days, Gahlot et al [5] found rate of CRBSI in their study it was $12.5 \%$ per 1000 catheter days. In the present study organisms isolated from CRBSI most predominantly belonged to family Enterobacteriaceae i.e. K.pneumoniae and E.cloacae (50\%) and 50\% isolates were yeasts i.e. C.albicans and Non albicans
Candida. Gaynesel al. [14] described that hands of health-care workers often introduce Gram-negative organisms during the manipulation of catheters or intravenous tubing.

None of the study to the best of our knowledge described Gram negative as most common organism in CRBSI but most of the studies have also discussed about the yeasts are also one of the most important cause of CRBSI.

Pawar et al. [13] and Subba Rao et al. [2] from Karnataka demonstrated that CRBSI was caused by Candida species in ICUs i.e. $11.4 \%$ and $20 \%$ respectively and have also showed that the commonest isolates in ICU patients were coagulase negative Staphylococcus (32.4\%), Pseudomonas and Enterobacter species. Parmeshawaram et al. [12] from Karnataka showed majority of the CRBSI were caused by Gram positive organisms i.e. $64 \%$.

Gahlot et al. from Varanasi demonstrated that out of the 14 CRBSI causing organisms two thirds were Gram positive cocci (71\%), 22\% were non-fermenters and 7\% cases were due to Candida species.

In the present study central line double lumen catheters were used and the site of insertion were subclavian vein, i.e. right subclavian vein $(68 \%)$ and left subclavian vein (32\%). Several variables have been quoted as contributing to catheter related blood stream infections, these include number of catheter lumens, cannulation sites, duration of catheterization and immune status of the patients. On the basis of discussion which cannulation site is associated with the higher risk of infection remains controversial. 
Original Research Article

The present study showed the highest incidence of local catheter infection at the subclavian site. Richet et al [15] from France explained that it could be related to the presence of hair in this area, contaminating with orophyngeal secretion, insertion technique, or jugular site dressings are often loose. Merrer et al. [16] and Goetz et al. [17] observed a higher incidence of infection related complications at the femoral venous site in comparison with subclavian vein site $(19.8 \%$ vs $4.5 \%)$.

Femoral access is often used in emergency situation during which adequate procedures cannot be always fully respected. Presence of higher density of local skin flora in the groin area is also postulated to be a reason.

In the present study, catheters were inserted for duration that ranged from 7 to 45 days with a mean of 21 days and a total of 1396 days. An increase in CVC colonization rate was observed with increasing duration of catheterization days, which is also supported by other studies like Gahlot et al from Varanasi.

Associated morbidities in the present study shows that $100 \%$ of the patients with CRBSI were having altered kidney functions and low haemoglobin count whereas $25 \%$ of them were having altered liver profile. Among the patients with catheter tip colonization $82 \%$ were having low haemoglobin and $77 \%$ were having altered kidney function and $34 \%$ were having altered liver function. Parmeshwaram et al from Karnataka also discussed that both the patients with both CRBSI and catheter tip colonization were having altered kidney profile i.e. $40 \%$ and $27.7 \%$ respectively.

S.marcescens showed 100\% sensitivity for Amoxicillin, Clavulenic acid, Ceftriaxone, Imipenem, Piperacilin Tazobactum, Tigecyclin and $100 \%$ resistance for Amikacin and Ciprofloxacin.

K. pneumoniae showed they were $100 \%$ resistant for Amikacin, Imipenem and Piperacillin-Tazobactum. E.cloacae were $100 \%$ sensitive for Amikacin, Ceftriaxone, Ciprofloxacin, Colistin, Imipenem and $60 \%$ were resistant for Amoxicillin clavulenic acid. $100 \%$ sensitivity for Tigecyclin and $67 \%$ sensitivity for Colistin.

Antibiogram profile of yeasts isolated from CRBSI were all of them were $100 \%$ sensitive to Amphotericin B, Caspofungin, Fluconazole and Variconazole. Whereas E.cloacae $100 \%$ sensitive to Amikacin, Ciprofloxacin, Ceftriaxone, Colistin and Imipenem and $40 \%$ resistance for Amoxicillin clavulanic acid. E. cloacae were $100 \%$ sensitive to Amikacin, Ceftriaxone, Ciprofloxacin, Colistin and Imipenem.

Due to limited resources the quantitative sonication method for the present study could not be utilized which would have proved better incidence of pathogens. Also, such type of studies can be carried out for a long duration so that exact incidence of pathogens and the risk factors associated could be known and measures can be taken to further reduce the number of cases.

\section{Conclusion}

Catheter colonization and duration of catheterization has an important role in development of CRBSI which may lead to septicaemia and multi-organ failure. CRBSI must be suspected in the catheterized patient having sign and symptoms of septicaemia. Local antibiotics and catheter lock solutions should be used as preventive measure while inserting the central line catheter.

\section{What the study adds to the existing knowledge?}

This study further provides evidence that after using a subclavian site, rather than a jugular or a femoral site, in adult patients, the risk of infection is minimized for non-tunneled CVC placement.

\section{Contributions from authors}

Dr. Nidhi Negi: Conceived and designed the analysis, Wrote the manuscript.

Ms Suchandra Gupta: Performed the analysis, collected data \& contributed to the Manuscript writing

Dr. Iva Chandola: Contributed to the Manuscript writing, Data analysis, reference checking

Prof V.K Kataria: Guided the research work as the senior-most member of the team.

Funding: No funding sources

Conflict of interest: None declared

Ethical Approval: This study was approved by the Institutional Ethics Committee

\section{References}

1. Mermel LA. Prevention of intravascular catheterrelated infections. Ann Intern Med. 2000;132(5):391402. doi: 10.7326/0003-4819-132-5-200003070-00009.

2. Subba Rao SD, Joseph MP, Lavi R, Macaden R. Infections related to vascular catheters in a pediatric intensive care unit. Indian Pediatr. 2005;42(7):667-672. 


\section{Original Research Article}

3. Orsini J, Mainardi C, Muzylo E, Karki N, Cohen N, Sakoulas G. Microbiological profile of organisms causing bloodstream infection in critically ill patients. J Clin Med Res. 2012;4(6):371-377. doi: 10.4021/jocmr 1099w. Epub 2012 Nov 11.

4. Hasan S, Imtiaz F, Khan M.A and Iqbal A. Blood stream infections and their sensitivity pattern in a tertiary care Hospital. 2015;2(52):2321-3485.

5. Gahlot R, Nigam C, Kumar V, Gupta M. Catheter related bloodstream infections in ICU: a study from North India. Int J Infection Control. 2013;9(2):4. doi: 10.3396/ijic.v9i2.013.13.

6. Lorente L, Henry C, Martín MM, Jiménez A, Mora ML. Central venous catheter-related infection in a prospective and observational study of 2,595 catheters. Critical Care. 2005;9(6):R631-R635.

7. Mermel LA, Farr BM, Sherertz RJ, Raad II, O'Grady $\mathrm{N}$, Harris JS, et al. Guidelines for the management of intravascular catheter-related infections. J Intraven Nurs. 2001;24(3):180-205.

8. Siegman-Igra Y, Anglim AM, Shapiro DE, Adal KA, Strain BA, Farr BM. Diagnosis of vascular catheterrelated bloodstream infection: a meta-analysis. J Clin Microbiol. 1997;35(4):928-936.

9.Maki DG, Weise CE, Sarafin HW. A semiquantitative culture method for identifying intravenous-catheterrelated infection. N Engl J Med. 1977;296 (23):13051309. doi: 10.1056/NEJM 197706092962301.

10. Sherertz RJ, Heard SO, Raad II. Diagnosis of triplelumen catheter infection: comparison of roll plate, sonication, and flushing methodologies. $\mathrm{J}$ Clin Microbiol. 1997; 35(3):641-646.
11. Garcia L.S, Isenberg H.D. Clinical Microbiology Procedures Handbook, 3rd edition, Volume-1. Catheter tip cultures 3.6.1-3.6.7.

12.Parameswaran R, Sherchan JB, Varma D M, Mukhopadhyay C, Vidyasagar S. Intravascular catheterrelated infections in an Indian tertiary care hospital. J Infect Dev Ctries. 2011;5(6):452-458.

13. Pawar M, Mehta Y, Kapoor P, Sharma J, Gupta A, Trehan N. Central venous catheter-related blood stream infections: incidence, risk factors, outcome, and associated pathogens. J Cardiothorac Vasc Anesth. 2004; 18(3):304-308. doi: 10.1053/j.jvca.2004.03.009.

14. Robert Gaynes. Definitions and epidemiology of nosocomial intravascular catheter-related (primary) bloodstream infections. 2009; 27.

15. Richet H, Hubert B, Nitemberg G, Andremont A, Buu-Hoi A, Ourbak P, et al. Prospective multicenter study of vascular-catheter-related complications and risk factors for positive central-catheter cultures in intensive care unit patients. J Clin Microbiol. 1990;28 (11): 2520-2525.

16. Merrer J, De Jonghe B, Golliot F, Lefrant JY, Raffy $\mathrm{B}$, Barre E, Complications of femoral and subclavian venous catheterization in critically ill patients: a randomized controlled trial. JAMA. 2001; 286(6):700707. doi: 10.1001/jama.286.6.700.

17. Goetz AM, Wagener MM, Miller JM, Muder RR. Risk of infection due to central venous catheters: effect of site of placement and catheter type. Infect Control Hosp Epidemiol. 1998;19(11):842-845. doi: https://doi. org / $10.2307 / 30141562$.

\section{How to cite this article?}

Negi N, Gupta S, Chandola I, Kataria V. Prevalence of central line associated blood stream infection (CRBSI) and catheter colonization in ICU settings of a tertiary care hospital in sub Himalayan region. Trop J Path Micro 2019; 5(10):815-821.doi:10.17511/jopm.2019.i10.10. 